\title{
Immunohistochemical analyses of the expression profiles of INSM1, ATRX, DAXX and DLL3 in solid papillary carcinomas of the breast
}

\author{
HIROTSUGU YANAI $^{1}$, MITSUAKI ISHIDA ${ }^{2}$, KATSUHIRO YOSHIKAWA ${ }^{1,2}$, \\ KOJI TSUTA ${ }^{2}$, MITSUGU SEKIMOTO ${ }^{1}$ and TOMOHARU SUGIE ${ }^{1}$ \\ Departments of ${ }^{1}$ Surgery, ${ }^{2}$ Pathology and Division of Diagnostic Pathology, \\ Kansai Medical University, Hirakata, Osaka 573-1191, Japan
}

Received December 26, 2021; Accepted February 22, 2022

DOI: $10.3892 / \mathrm{ol} .2022 .13257$

\begin{abstract}
Solid papillary carcinoma (SPC) is a rare but distinct clinicopathological feature of breast cancer characterised by frequent neuroendocrine differentiation. Insulinoma-associated protein 1 (INSM1) is a useful neuroendocrine marker for various neuroendocrine tumours. $\alpha$-thalassemia/mental retardation syndrome X-linked protein (ATRX) and death domain-associated protein (DAXX) are useful prognostic markers for patients with pancreatic neuroendocrine tumours. However, to the best of our knowledge, few studies have addressed INSM1 expression in SPCs. Although ATRX, DAXX and $\delta$-like canonical notch ligand 3 (DLL3) are frequently expressed in neuroendocrine lung carcinomas, there are no reports on their expression in SPCs. Therefore, the present study aimed to analyse the expression profiles of INSM1, ATRX, DAXX and DLL3 in the largest series of patients with SPC that has been, to the best of our knowledge, studied until now. Immunohistochemical analyses were performed to determine chromogranin A, synaptophysin, INSM1, ATRX, DAXX and DLL3 expression in 39 specimens surgically resected from patients with SPC (18 SPC in situ and 21 SPC invasive). The associations between the expression of these markers and the clinicopathological factors were investigated. Chromogranin A, synaptophysin
\end{abstract}

Correspondence to: Dr Mitsuaki Ishida, Department of Pathology and Division of Diagnostic Pathology, Kansai Medical University, 2-3-1 Shinmachi, Hirakata, Osaka 573-1191, Japan

E-mail: ishidamt@hirakata.kmu.ac.jp

Abbreviations: ATRX, $\alpha$-thalassemia/mental retardation syndrome X-linked protein; DAXX, death domain-associated protein; DLL3, $\delta$-like canonical notch ligand 3; INSM1, insulinoma-associated protein 1; LI, labelling index; SPC, solid papillary carcinoma

Key words: solid papillary carcinoma, breast carcinoma, neuroendocrine differentiation, insulinoma-associated protein 1 , $\alpha$-thalassemia/mental retardation syndrome X-linked protein, death domain-associated protein, $\delta$-like canonical notch ligand 3 and INSM1 were expressed in 64.1, 100 and 92.3\% of the patients, respectively. Both ATRX and DAXX expression was observed in $28.2 \%$ of the patients. No patient expressed DLL3. Lack of INSM1 or chromogranin A expression was significantly associated with advanced pathological stages in patients with SPC $(\mathrm{P}=0.033)$ and in patients with invasive SPC $(\mathrm{P}=0.012)$, showing a tendency for a high Ki-67 labelling index (LI) and advanced histological grade in patients with invasive SPC. Loss of ATRX or DAXX expression was significantly associated with lymphatic invasion, but not with histological grade, Ki-67 LI or presence of invasive tumours. Thus, INSM1 was demonstrated to be a useful diagnostic marker for SPCs. Overall, detecting the lack of INSM1 or chromogranin A expression may be useful for analysing the characteristics of tumour cells in SPCs.

\section{Introduction}

Solid papillary carcinoma (SPC) is a relatively rare but distinct clinicopathological feature of breast carcinomas with frequent neuroendocrine differentiation, accounting for $\sim 1 \%$ of all breast carcinomas $(1,2)$. Histopathologically, it is characterised by solid and/or papillary growth patterns with delicate fibrovascular stroma, and it may be classified as either SPC in situ (absence of invasive lesions) or SPC invasive (presence of invasive lesions) $(1,2)$. It has been established that SPCs frequently undergo neuroendocrine differentiation detected by immunohistochemical analyses of classical neuroendocrine markers, such as chromogranin A and synaptophysin $(1,2)$.

Lately, some novel neuroendocrine markers have been used for routine histopathological diagnosis, and insulinoma-associated protein 1 (INSM1) is one of the most notable markers. Incidentally, INSMI was originally identified from a subtraction library of insulinoma. It encodes a transcription factor with five zinc-finger motifs, essential for developing neuroendocrine cells $(3,4)$. The INSMI is predominantly expressed in the developing neuroendocrine tissues, and it is activated by neurogenin 3 , following NeuroD/ $\beta 2$ activation in pancreatic endocrine cells (5). Expression of INSM1 is suppressed by the Notch1-HES1 signalling pathway, thereby repressing neuroendocrine differentiation (6). Subsequently, 
its expression has been reported in various neuroendocrine tumours, such as pituitary adenoma (7), medullary carcinoma of the thyroid $(7,8)$, carcinoid tumour, small and large cell neuroendocrine carcinoma of the lung (9-11), neuroendocrine carcinoma of the gastrointestinal tract (12), and pancreatic neuroendocrine tumours $(12,13)$. Additionally, excellent sensitivity and specificity of INSM1 for neuroendocrine differentiation in these tumours have also been described (7-13). To date, the expression of INSM1 in SPC has been analysed only in three reports (14-16), with the largest case series comprising of 19 patients, which showed that INSM1 is a useful neuroendocrine marker in SPC because 58\% (11 of 19 patients) showed positive immunoreactivity (14).

The genes for $\alpha$-thalassemia/mental retardation syndrome $\mathrm{X}$-linked protein $(A T R X)$ and death domain-associated protein $(D A X X)$ play important roles in chromatin remodelling at the telomeres and other genomic sites in normal tissues; moreover, whole-exome analysis has revealed that inactivated somatic mutations of these genes are frequently observed in pancreatic neuroendocrine tumours (17). Subsequent studies have revealed that loss of ATRX and DAXX is associated with chromosomal instability and a shorter survival period in patients with pancreatic neuroendocrine tumours $(18,19)$. However, the frequency of loss of ATRX and DAXX expression and its prognostic significance in SPC patients has not yet been analysed. Moreover, $\delta$-like canonical Notch ligand 3 (DLL3), which plays an important role in Notch signalling, is frequently expressed in neuroendocrine-related tumours, such as neuroendocrine carcinomas of the lungs (20). However, DLL-3 expression and its prognostic significance in SPC have not yet been analysed. Therefore, the present study aimed to analyse the immunohistochemical expression profiles of INSM1, ATRX, DAXX, and DLL-3 in the largest series of patients with SPC of the breast studied to date.

\section{Materials and methods}

Patient selection. We selected 39 patients with SPC, based on the recent World Health Organization Classification of Breast Tumours (1). The patients had consecutively undergone surgical resections at the Department of Surgery of the Kansai Medical University Hospital between January 2006 and December 2020.

This retrospective single-institution study was conducted following the principles of the Declaration of Helsinki, and the study protocol was approved by the Institutional Review Board of the Kansai Medical University Hospital (approval \#2019059). All data are completely anonymised. The Institutional Review Board waived the requirement for informed consent due to the retrospective design of the study, using medical records and archival samples without risk of identity exposure to the patients. Moreover, the present study did not include any minors. All information regarding this study, such as the inclusion criteria and the opportunity to opt out, are provided through the institutional website (https://www.kmu.ac.jp/hirakata/ hospital/2671t800000136cd-att/a1642569623481.pdf).

Histopathological analysis. Surgically resected specimens were fixed with $10 \%$ formalin at room temperature (24-48 h), sectioned $(5-10 \mathrm{~mm})$, dehydrated by ethanol and xylene at room temperature, embedded in paraffin $\left(60^{\circ} \mathrm{C}\right)$, and stained with haematoxylin and eosin (5 min each) at room temperature. More than two experienced pathologists independently evaluated the histopathological features. We used the TNM Classification of Malignant Tumours, Eighth Edition. The histopathological grading of the invasive tumours was based on the Nottingham histological grade (21). The Ki-67 labelling index (LI) of invasive tumours was considered high when $>20 \%$ of the neoplastic cells were labelled because the median Ki-67 LI for invasive carcinomas of no special type in our institute is $20 \%$.

Immunohistochemistry. Immunohistochemical analyses of 4-micrometer sections of whole tumour tissues containing in situ and/or invasive regions were performed using autostainers (Ultra System, Roche Diagnostics, or Autostainer link 48, DakoCytomation), according to the manufacturer's instructions. Rabbit primary polyclonal antibody against ATRX (HPA001906; Atlas Antibodies; diluted 1:800; incubation time $20 \mathrm{~min}$ at room temperature), mouse monoclonal antibody against chromogranin A (LK2H10; Cell Marque; diluted 1:200; incubation time $20 \mathrm{~min}$ at room temperature), rabbit polyclonal antibody against DAXX (HPA008736; Atlas Antibodies; diluted 1:250; incubation time $20 \mathrm{~min}$ at room temperature), rabbit monoclonal antibody against DLL3 (SP347; Roche Diagnostics; prediluted; incubation time 20 min at room temperature), mouse monoclonal antibody against INSM1 (A8; Santa Cruz Biotechnology; diluted 1:50; incubation time $30 \mathrm{~min}$ at room temperature), and mouse monoclonal antibody against synaptophysin (27G12; Nichirei Bioscience; pre-diluted; incubation time $20 \mathrm{~min}$ at room temperature) were used. Secondary antibody was pre-diluted $\left(37^{\circ} \mathrm{C}\right)$ [OptiView DAB Universal Kit (cat. no. 518-111427; Roche)]. At least two researchers independently evaluated the immunohistochemical staining of the tumours using the microscope (Olympus BX53). Any tumour with $>1 \%$ of neoplastic cells showing positive immunoreactivity for chromogranin A, synaptophysin, INSM1, and DLL3 was recognised as a positive tumour. All tumour cells expressing ATRX or DAXX were considered ATRX- or DAXX-retained, respectively, and the ones without ATRX and/or DAXX were considered negative cells, i.e., they had undergone loss of these markers.

Statistical analyses. All statistical analyses were performed using JMP Start Statistics version 14.2 (Statistical Discovery Software; SAS Institute). Associations between the two groups were evaluated using Fisher's exact test for categorical variables and Wilcoxon's rank test for continuous variables. $\mathrm{P}<0.05$ was considered to indicate a statistically significant difference.

\section{Results}

Patient characteristics and immunohistochemical analyses. Table I summarises the clinicopathological features of the study cohort. This study comprised 39 female patients with SPC. At the initial diagnosis, the median age was 72 years (age range: 39-87 years). The cohort included 18 patients with SPC in situ (pathological stage 0) and 21 patients with SPC invasive. Typical histopathological features of SPC in situ and SPC invasive are shown in Fig. 1A and B, 
Table I. Clinicopathological characteristics of patients with SPC.

\section{Factors}

n

Total number of subjects

39

Median age, years (range)

$72.0(39-87)$

Menopausal status

Premenopausal

10.3

Postmenopausal

4

87.2

Unknown

34

2.6

Median body mass index, $\mathrm{kg} / \mathrm{m}^{2}$ (range)

Median tumor size of the invasive component, $\mathrm{mm}$ (range)

$23.8(16.4-34.9)$

$1(0-45)$

Pathological stage

0

I

IIA

15

38.5

IIB

4

10.3

IIIA

2

5.1

IIIB

0

0.0

IIIC

Lymph node status

Positive

Negative

Not tested

Lymphatic invasion

Positive

Negative

Venous invasion

Positive

Negative

Nottingham histological grade (SPC invasive)

Ki-67 labeling index (SPC invasive)

$>20 \%$

$\leq 20 \%$

Estrogen receptor

Positive

Negative

Progesterone receptor

Positive

Negative

Human epidermal growth factor receptor 2

Positive

Negative

Synaptophysin

Positive

Negative

Chromogranin A

Positive

Negative

Insulinoma-associated protein 1 
Table I. Continued.

Factors

$\mathrm{n}$

A-thalassemia/mental retardation syndrome $\mathrm{X}$-linked protein

Retained

28.2

Lost

Death domain associated protein

Retained

Lost

$\Delta$-like canonical Notch ligand 3

Positive

Negative

Recurrence

No recurrence

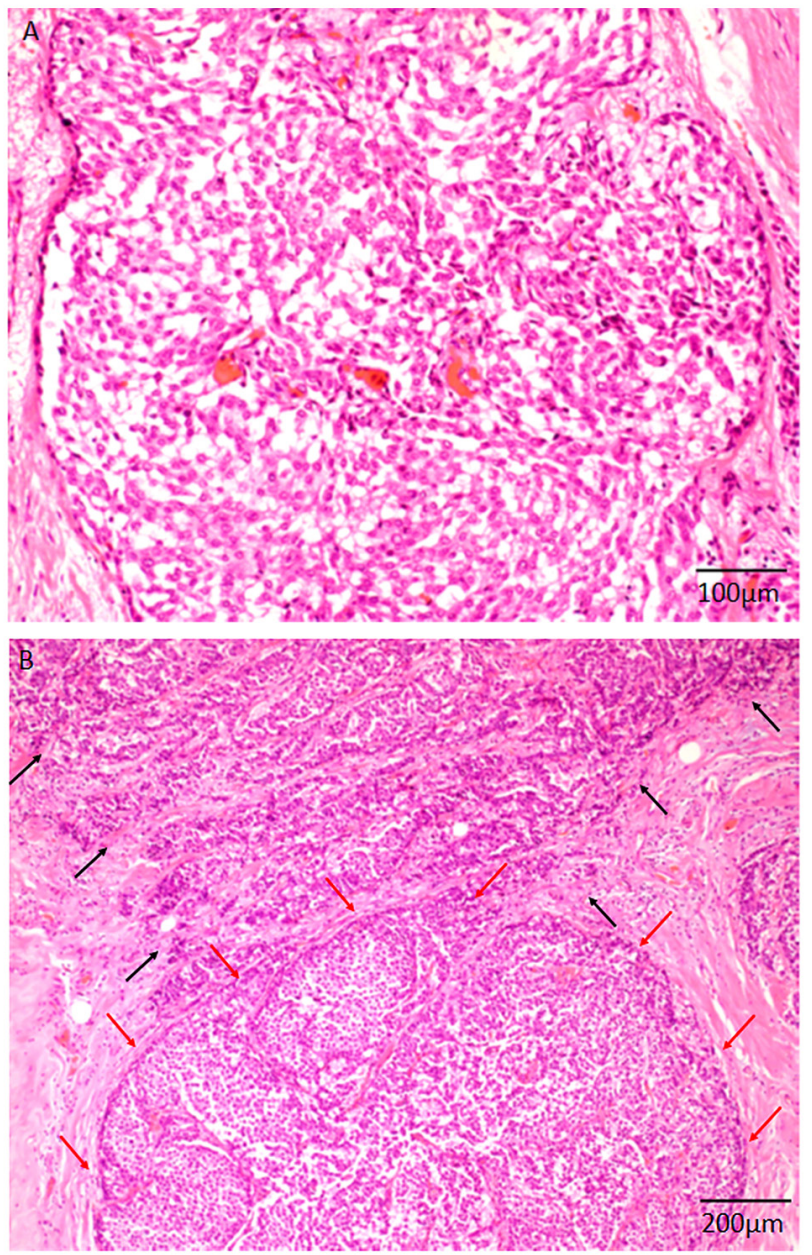

Figure 1. Histopathological features of solid papillary carcinoma. (A) Solid papillary carcinoma in situ. Intraductal solid proliferation of the neoplastic cells with slightly enlarged round nuclei and eosinophilic cytoplasm accompanying delicate fibrovascular core (haematoxylin and eosin stain; magnification, x200; scale bar, $100 \mu \mathrm{m}$ ) can be observed. (B) Invasive solid papillary carcinoma. Infiltrative neoplastic growths forming irregular nests (upper, black arrows) and in situ lesions (lower, red arrows) can be noted. These neoplastic cells have slightly enlarged round nuclei and eosinophilic cytoplasm (haematoxylin and eosin stain; magnification, x100; scale bar, $200 \mu \mathrm{m})$. respectively. SPC in situ composed of intraductal solid proliferation of the neoplastic cells with slightly enlarged round nuclei and eosinophilic cytoplasm (Fig. 1A). Both infiltrative neoplastic growths forming irregular nests and in situ lesions were noted in SPC invasive (Fig. 1B). Among the 18 patients with SPC in situ, 15 had not undergone lymph node resection. Moreover, the patients with SPC in situ were not tested for human epidermal growth factor receptor 2 (HER2), because these patients were not the possible candidate for anti-HER 2 therapy. Recurrence, particularly bone metastasis, was observed in only one patient with invasive SPC. The median follow-up period was 33 months (range: 1-152 months). Table I summarises the results of the immunohistochemical analyses of the tumour specimens. All the tumour specimens tested positive for oestrogen and progesterone receptors, except for one patient in the SPC invasive group. Interestingly, HER2 was not overexpressed in any of the samples from the SPC invasive group. Chromogranin A was expressed in $64.1 \%$ (25 out of 39) of in situ and invasive lesions (Fig. 2A and B), whereas synaptophysin was expressed in all samples of in situ and invasive lesions (Fig. 2C and D). INSM1 expression was noted in $92.3 \%$ (36 out of 39) of in situ and some of the invasive lesions (Fig. 3A and B). Each of ATRX and DAXX expression was retained in 28.2\% (11 out of 39) of in situ lesions, and some invasive neoplastic cells showed loss of nuclear immunoreactivity of ATRX or DAXX (Fig. 3C-F), whereas none of the patients showed positive immunoreactivity for DLL3 (Fig. 3G).

Association between clinicopathological factors and the expression of chromogranin A, synaptophysin, and INSMI. Table II summarises the associations between the clinicopathological factors observed in patients with SPC and the expression of chromogranin A, synaptophysin, and INSM1. The distribution of these three markers is shown in Fig. 4. Among the 39 patients, 23 showed positive immunoreactivity for all three markers, while the remaining 16 lacked 


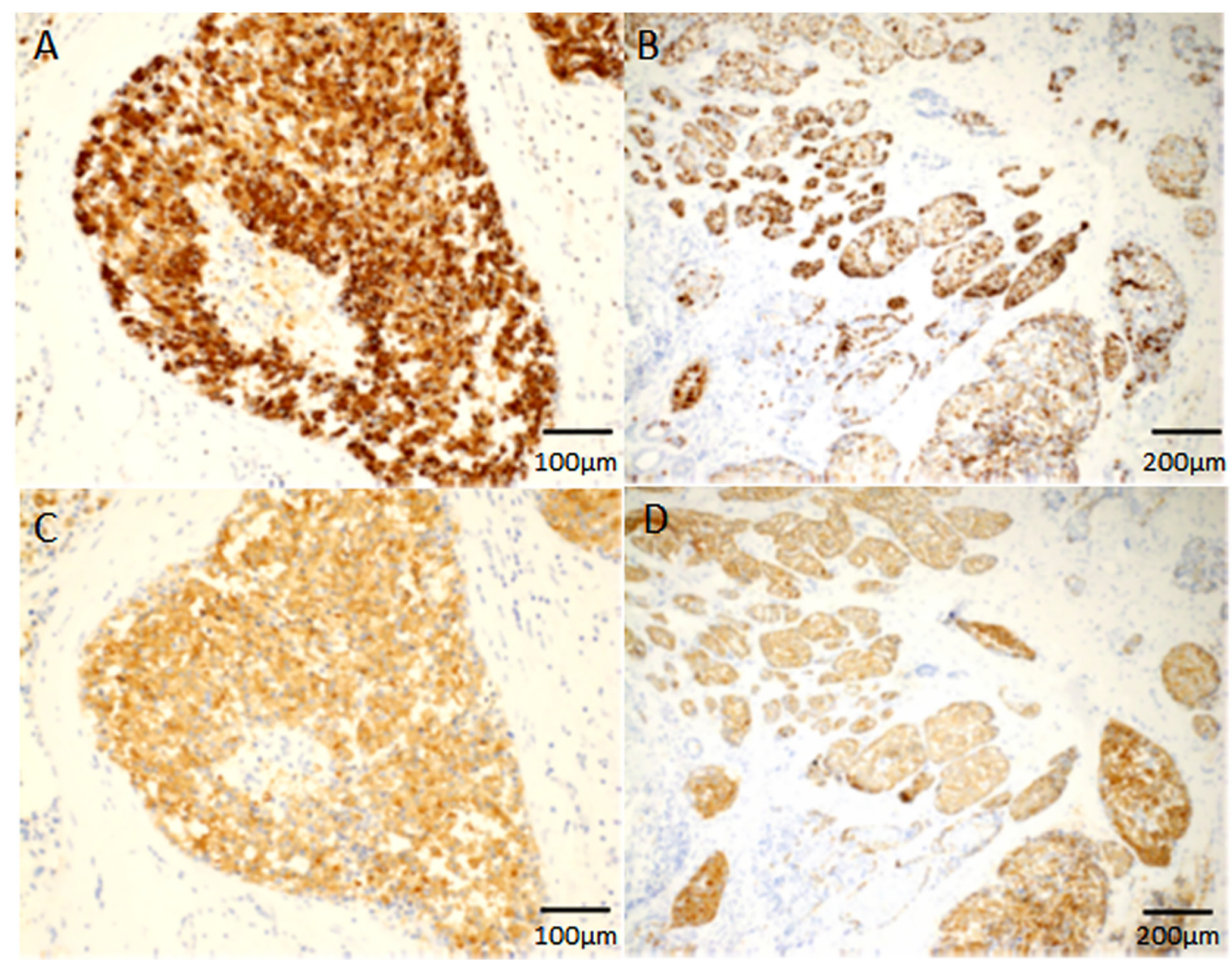

Figure 2. Chromogranin A and synaptophysin expression in solid papillary carcinoma. Chromogranin A is expressed in the cytoplasm of neoplastic cells in the (A) in situ (magnification, x200; scale bar, $100 \mu \mathrm{m}$ ) and the (B) invasive lesions (magnification, $\mathrm{x} 100$; scale bar, $200 \mu \mathrm{m}$ ). Synaptophysin is expressed in the cytoplasm of neoplastic cells in the (C) in situ (magnification, x200; scale bar, $100 \mu \mathrm{m}$ ) and the (D) invasive lesions (magnification, x100; scale bar, $200 \mu \mathrm{m}$ ).

expression of more than one marker (Fig. 4). The advanced pathological stages and body mass index were significantly correlated with the lack of expression of either INSM1 or chromogranin A ( $\mathrm{P}=0.033$ and 0.049 , respectively). By contrast, other clinicopathological factors, including the presence of invasive tumours, were not significantly correlated with the lack of expression of INSM1 or chromogranin A. Moreover, the sample from only patient who showed recurrence tested negative for chromogranin A and INSM1.

Table III summarises the associations between the clinicopathological factors of the patients in the SPC invasive group and the expression of chromogranin $\mathrm{A}$, synaptophysin, and INSM1. Incidentally, the lack of chromogranin A or INSM1 expression in the invasive component was significantly associated with a higher pathological stage $(\mathrm{P}=0.012)$ and a tendency for advanced histological grade and high Ki-67 LI, but the difference was not significant $(\mathrm{P}=0.090$ and 0.064 , respectively).

Association between clinicopathological factors and the expression of ATRX and DAXX. Table IV summarises the association between the clinicopathological factors of the patients with SPC and the expression of ATRX and DAXX. Among the 39 patients, samples from eight had retained both ATRX and DAXX expression, while those of the remaining 31 showed loss of ATRX and/or DAXX expression. Although the median age of the patients with loss of ATRX and/or DAXX was significantly higher than that of the patients who had retained the expression of ATRX and DAXX, there were no significant differences between the groups, even for the presence of invasive tumours. Moreover, the only patient to show recurrence exhibited loss of ATRX and/or DAXX.

Table V summarises the association between the clinicopathological factors of the patients in the SPC invasive group and ATRX and DAXX expression. Among the 21 patients, nine had retained both ATRX and DAXX expression in the invasive component, while the remaining 12 showed loss of ATRX and/or DAXX expression in the invasive component. The presence of lymphatic invasion was significantly correlated with the loss of ATRX and/or DAXX expression $(\mathrm{P}=0.019)$. However, the Nottingham histological grade and Ki-67 LI were not significantly different between the two groups.

\section{Discussion}

In the present study, we demonstrated that INSM1 is a useful neuroendocrine marker for diagnosing breast SPC, and it might even be superior to chromogranin A (positive rates were 92.3 and $64.1 \%$ for INSM1 and chromogranin A, respectively). Additionally, the lack of INSM1 or chromogranin A expression was significantly correlated with an advanced pathological 


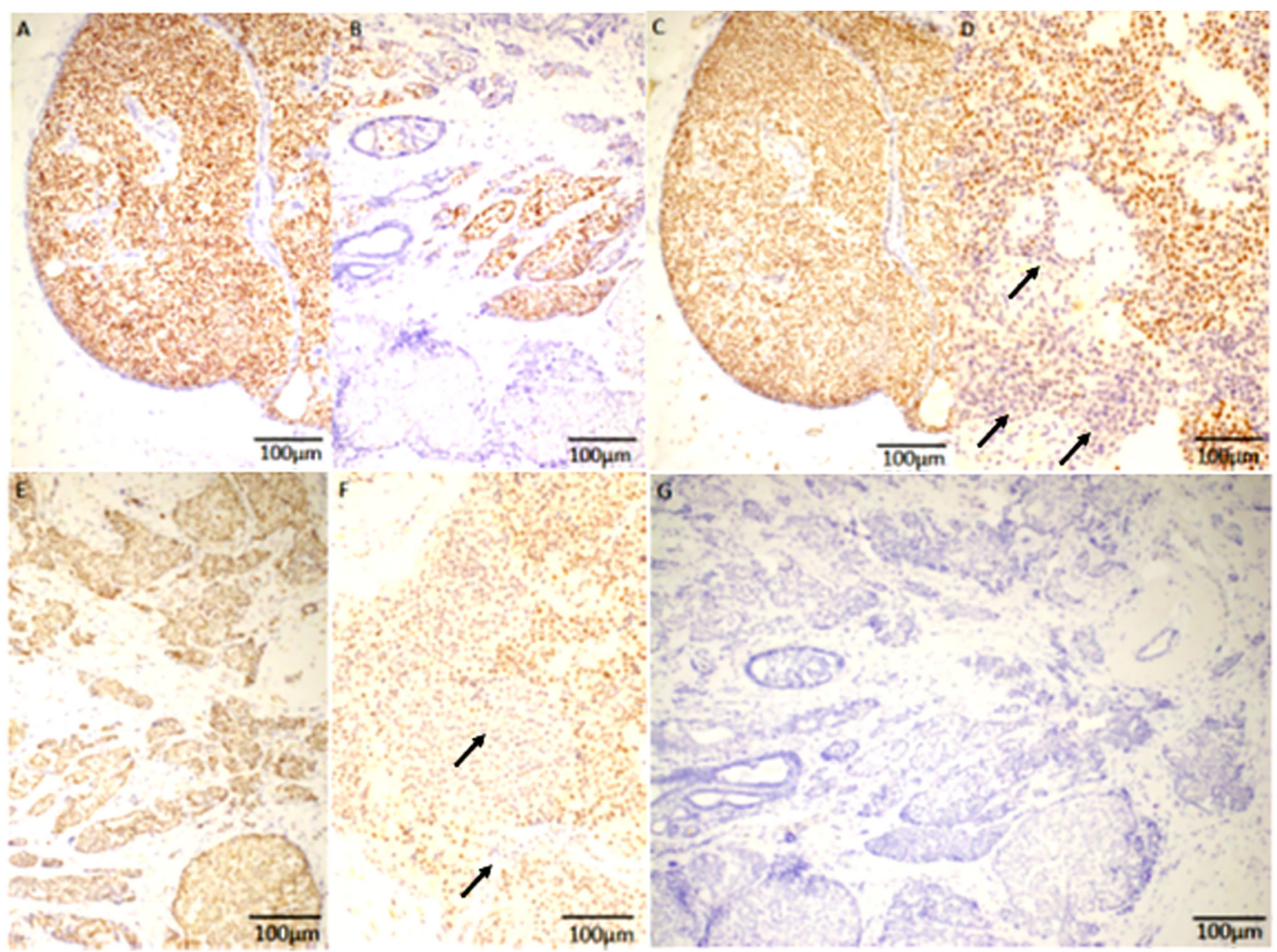

Figure 3. INSM1, ATRX, DAXX and DLL3 expression in SPC. (A) Diffuse nuclear expression of INSM1 is noted in SPC in situ. (B) Some invasive neoplastic cells show positive nuclear immunoreactivity (brown stain) for INSM1. (C) Retained nuclear expression of ATRX is noted in the SPC in situ group. (D) Some invasive neoplastic cells show loss of nuclear immunoreactivity for ATRX (arrows). (E) Retained nuclear expression of DAXX is noted in the SPC invasive group. (F) Some of the invasive neoplastic cells show loss of nuclear immunoreactivity for DAXX (arrows). (G) None of the samples suggested positive expression for DLL3. Magnification, x200; scale bars, $100 \mu \mathrm{m}$. INSM1, Insulinoma-associated protein 1; DAXX, death domain associated protein; ATRX, $\alpha$-thalassemia/mental retardation syndrome X-linked protein; SPC, solid papillary carcinoma; DLL3, $\delta$-like canonical notch ligand 3; SPC, solid papillary carcinoma.

stage in patients with SPC, and in patients with invasive SPC, it showed a significant association of a higher pathological stage and a tendency to be associated with high Ki-67 LI and advanced histological grade. Moreover, the loss of ATRX or DAXX expression was significantly correlated with the presence of lymphatic invasion.

Incidentally, INSM1 has been recognised as a useful and specific neuroendocrine marker because it is an essential transcription factor for developing normal neuroendocrine cells wherein its expression is highly restricted (3-5). Previously several reports have concluded that INSM1 expression is specific to various neuroendocrine tumours (7-13). Furthermore, SPC is a distinct clinicopathological feature of breast carcinoma that frequently shows neuroendocrine differentiation; SPC shows frequent positive immunoreactivity for chromogranin A and synaptophysin $(1,2)$. However, to the best of our knowledge, only three studies have addressed the expression of INSM1 in SPCs (14-16). According to a previous report, INSM1 expression was noted in 11 out of 19 patients with SPC (2 of 3 SPC in situ, and nine of 16 SPC invasive). Among these 19 patients with SPC, 11 tested positive for synaptophysin and/or chromogranin A, while the remaining eight were synaptophysin- and chromogranin A-negative. Positive INSM1 expression was noted in nine of the 11 above-mentioned neuroendocrine marker-positive patients and two of the eight neuroendocrine marker-negative patients (14). Additionally, another report showed that eight out of eight (including one patient without synaptophysin and chromogranin A expression) patients showed positive INSM1 expression (15) (INSM1 expression rate in SPC was not available in the remaining report (16)). The current study includes the analysis of the largest series of SPCs for INSM1 expression, and it shows that $92.3 \%$ (36 of 39) of the patients tested positive for INSM1 expression. According to these results, it may be suggested that although INSM1 is a useful neuroendocrine marker, neuroendocrine differentiation in SPCs might not be defined by only INSM1 (14). Therefore, a combination of classical neuroendocrine markers and INSM1 might be useful for detecting neuroendocrine differentiation in SPCs because INSM1 may be expressed in patients with luminal B breast cancer, even if samples test negative for chromogranin A and synaptophysin (22). Moreover, this 
Table II. Associations between clinicopathological factors and the expressions of chromogranin A, synaptophysin and INSM1.

\begin{tabular}{|c|c|c|c|}
\hline Factors & $\begin{array}{l}\text { Chromogranin A, synaptophysin, and } \\
\text { INSM1-positive }(\mathrm{n}=23)\end{array}$ & $\begin{array}{l}\text { Chromogranin A, synaptophysin, or } \\
\text { INSM1-negative }(\mathrm{n}=16)\end{array}$ & P-value \\
\hline Median age, years \pm SD & $70.0 \pm 13.7$ & $72.0 \pm 9.1$ & 0.764 \\
\hline Menopausal status & & & 0.124 \\
\hline Premenopausal & 4 & 0 & \\
\hline Postmenopausal & 18 & 16 & \\
\hline Unknown & 1 & 0 & \\
\hline Median body mass index, $\mathrm{kg} / \mathrm{m}^{2} \pm \mathrm{SD}$ & $24.3 \pm 4.1$ & $23.1 \pm 2.9$ & 0.049 \\
\hline SPC in situ or invasive & & & 0.516 \\
\hline SPC invasive & 11 & 10 & \\
\hline SPC in situ & 12 & 6 & \\
\hline Pathological stage & & & 0.033 \\
\hline $0+\mathrm{I}$ & 22 & 11 & \\
\hline II & 1 & 5 & \\
\hline Lymph node status & & & $>0.999$ \\
\hline Positive & 1 & 2 & \\
\hline Negative & 11 & 10 & \\
\hline Not tested & 11 & 4 & \\
\hline Estrogen receptor & & & $>0.999$ \\
\hline Positive & 22 & 16 & \\
\hline Negative & 1 & 0 & \\
\hline Progesterone receptor & & & $>0.999$ \\
\hline Positive & 22 & 16 & \\
\hline Negative & 1 & 0 & \\
\hline
\end{tabular}

INSM1, insulinoma-associated protein 1; SPC, solid papillary carcinoma.

study demonstrated for the first time that the lack of INSM1 or chromogranin A expression is significantly correlated with an advanced pathological stage in patients with SPC, and it is associated with a significant higher pathological stage and a tendency toward high Ki-67 LI and advanced histological grade in patients with invasive SPC. Hence, examining INSM1, chromogranin A, and synaptophysin expression might be useful for analysing the characteristics of tumour cells in SPCs. A previous study demonstrated that NOTCH 1 expression was absent in SPC (14), which corresponded to the fact that INSM1 is essential for neuroendocrine differentiation, and Notch 1 negatively regulates neuroendocrine differentiation $(6,14)$. Moreover, DLL3 plays an important role and is considered an autonomous inhibitor of Notch signalling, and SPCs show positive immunoreactivity for Notch 2 and Notch $3(14,20)$. DLL3 expression was not detected in all patients with SPC in the present cohort, consistent with the results of a previous study (23). Although the detailed mechanism regarding the relationship among DLL3, Notch 2, and Notch 3 expression is unknown, lack of DLL3 expression in SPC might be related to the expression of Notch 2 and Notch 3.

If the expression of ATRX and DAXX is retained, then the prognosis of patients with pancreatic neuroendocrine tumours is improved $(18,19)$. This study is the first to address the expression of these two markers in patients with SPC.
However, in the present cohort, we observed that the loss of ATRX or DAXX expression is significantly correlated with the presence of lymphatic invasion, but not with an advanced histological grade or a high Ki-67 LI. Loss of ATRX or DAXX expression is associated with chromosomal instability and poor survival in patients with pancreatic neuroendocrine tumours (18). Therefore, a significant presence of lymphatic invasion in the present cohort might be related to poor survival in pancreatic neuroendocrine tumours (18). However, the present study did not analyse the prognosis of patients.

There are several limitations to the present study. First, this study was a retrospective single-centre analysis, which could have led to a selection bias; however, this is the largest series of patients with SPC studied to date. Second, this study included only one patient with recurrence, even though SPC is recognised as an indolent breast cancer and metastasis and/ or recurrence is relatively rare (1). Therefore, the prognostic significance of INSM1, ATRX, and DAXX expression is difficult to determine. Third, synaptophysin was expressed in all patients with SPC in the present cohort. However, synaptophysin expression may sometimes be lost $(2,14)$, and the rate of INSM1 expression is different between synaptophysin- and/ or chromogranin A-positive and synaptophysin- and chromogranin A-negative SPC patients (14). Hence, the expression of ATRX and DAXX might be different between these two 
Table III. Associations between the clinicopathological factors of patients in the solid papillary carcinoma invasive group and expression of chromogranin A, synaptophysin, and INSM1.

\begin{tabular}{lccr}
\hline Factors & $\begin{array}{c}\text { Chromogranin A, synaptophysin, and } \\
\text { INSM1-positive (n=10) }\end{array}$ & $\begin{array}{c}\text { Chromogranin A, synaptophysin, or } \\
\text { INSM1-negative (n=11) }\end{array}$ & P-value \\
\hline Pathological stage & 10 & 5 & 0.012 \\
I & 0 & 6 & 0.149 \\
II & 1 & 5 & $>0.999$ \\
Lymphatic invasion & 9 & 6 & 0.090 \\
Positive & & & \\
Negative & 1 & 2 & 0.064 \\
Venous invasion & 9 & 9 & \\
Positive & & & \\
Negative & 10 & 7 & \\
Nottingham histological grade & 0 & 4 & \\
1+2 & & 6 & \\
3 & 1 & 5 & \\
Ki-67 labelling index $(\%)$ & 9 & & \\
High $(>20)$ & & & \\
Low $(\leq 20)$ & & & \\
\hline
\end{tabular}

INSM1, insulinoma-associated protein 1.

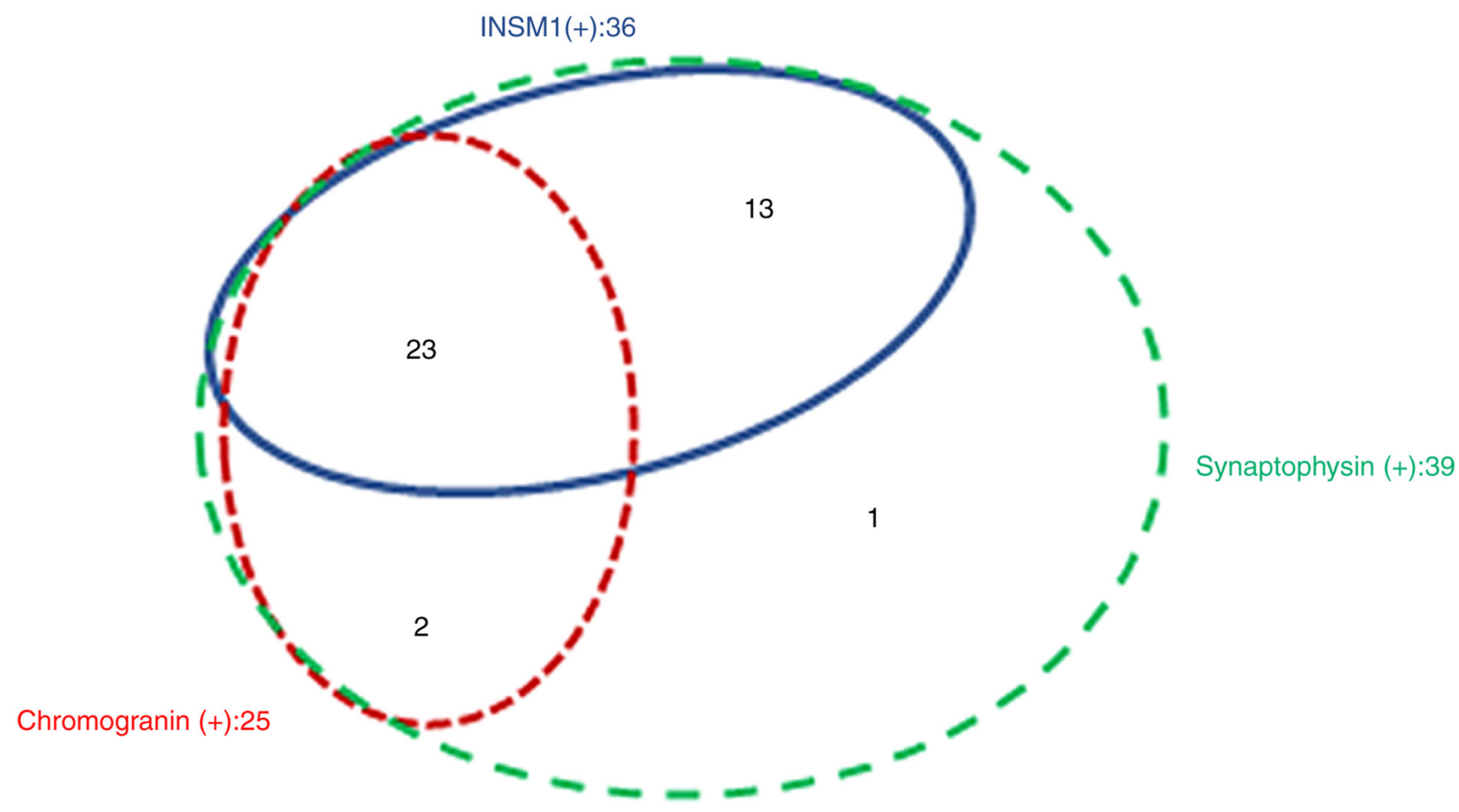

Figure 4. Venn diagram showing the distribution of chromogranin A, synaptophysin and INSM1 expression in solid papillary carcinoma. INSM1, Insulinoma-associated protein 1.

groups. Accordingly, additional studies must be performed with larger series of patients with SPC than the present study, who may/may not show metastasis and may test positive or negative for chromogranin A and/or synaptophysin. This will help analyse the clinical significance of the expression of these markers.
In conclusion, we studied the largest series of SPCs to date and demonstrated that INSM1 is a useful neuroendocrine marker for SPCs of the breast, and it might even be superior to chromogranin A. Furthermore, the lack of INSM1 or chromogranin A expression is significantly correlated with advanced pathological stages in patients with SPC. There is a significant 
Table IV. Association between clinicopathological factors and the expressions of ATRX and DAXX.

\begin{tabular}{|c|c|c|c|}
\hline Factors & ATRX- and DAXX-retained $(n=8)$ & ATRX- and/or DAXX-loss $(n=31)$ & P-value \\
\hline Median age, years \pm SD & $62.5 \pm 13.9$ & $72.0 \pm 10.3$ & 0.016 \\
\hline Menopausal status & & & 0.189 \\
\hline Premenopausal & 2 & 2 & \\
\hline Postmenopausal & 6 & 28 & \\
\hline Unknown & 0 & 1 & \\
\hline Median body mass index, $\mathrm{kg} / \mathrm{m}^{2} \pm \mathrm{SD}$ & $24.0 \pm 3.8$ & $23.8 \pm 3.9$ & 0.702 \\
\hline SPC in situ or invasive & & & 0.702 \\
\hline SPC invasive & 5 & 16 & \\
\hline SPC in situ & 3 & 15 & \\
\hline Pathological stage & & & $>0.999$ \\
\hline $0+\mathrm{I}$ & 7 & 26 & \\
\hline II & 1 & 5 & \\
\hline Lymph node status & & & $>0.999$ \\
\hline Positive & 1 & 2 & \\
\hline Negative & 5 & 16 & \\
\hline Not tested & 2 & 13 & \\
\hline Estrogen receptor & & & 0.205 \\
\hline Positive & 7 & 31 & \\
\hline Negative & 1 & 0 & \\
\hline Progesterone receptor & & & 0.205 \\
\hline Positive & 7 & 31 & \\
\hline Negative & 1 & 0 & \\
\hline
\end{tabular}

DAXX, death domain associated protein; ATRX, $\alpha$-thalassemia/mental retardation syndrome X-linked protein; SPC, solid papillary carcinoma.

Table V. Association between clinicopathological factors of patients in the SPC invasive group and the expression of ATRX and DAXX.

\begin{tabular}{|c|c|c|c|}
\hline Factors & ATRX- and DAXX-retained $(n=9)$ & ATRX- and/or DAXX-loss $(n=12)$ & P-value \\
\hline Lymphatic invasion & & & 0.019 \\
\hline Positive & 0 & 6 & \\
\hline Negative & 9 & 6 & \\
\hline \multicolumn{4}{|l|}{ Venous invasion } \\
\hline Positive & 1 & 2 & $>0.999$ \\
\hline Negative & 8 & 10 & \\
\hline \multicolumn{4}{|c|}{ Nottingham histological grade } \\
\hline $1+2$ & 9 & 8 & 0.104 \\
\hline 3 & 0 & 4 & \\
\hline \multicolumn{4}{|c|}{ Ki-67 labeling index $(\%)$} \\
\hline High $(>20)$ & 2 & 5 & 0.642 \\
\hline Low $(\leq 20)$ & 7 & 7 & \\
\hline
\end{tabular}

DAXX, death domain associated protein; ATRX, $\alpha$-thalassemia/mental retardation syndrome X-linked protein; SPC, solid papillary carcinoma.

association between a higher pathological stage and a tendency toward high Ki-67 LI and an advanced stage histological grade in patients with invasive SPC. However, loss of ATRX or DAXX expression, both of which are useful prognostic markers for patients with pancreatic neuroendocrine tumours, is significantly correlated with the presence of lymphatic invasion. Additional studies with larger cohorts are needed to determine the clinical significance of the expression of these markers. 


\section{Acknowledgements}

Not applicable.

\section{Funding}

No funding was received.

\section{Availability of data and materials}

All data generated and analysed in the present study are included in this published article.

\section{Authors' contributions}

HY and MI conceived and designed the study and performed the immunohistochemical analyses. HY, MI, KY, KT, MS and TS acquired and analysed the data. HY and MI confirmed the authenticity of all the raw data. HY and MI drafted the manuscript, tables and figures. All authors have read and approved the final manuscript.

\section{Ethics approval and consent to participate}

This retrospective single-institution study was conducted in accordance with the principles of the Declaration of Helsinki, and the study protocol was approved by the Institutional Review Board of the Kansai Medical University Hospital (approval no. 2019059; Hirakata, Japan). All data are completely anonymized. The Institutional Review Board waived the requirement of informed consent due to the retrospective design of the study, using medical records and archival samples with no risk of identity exposure for the patients. Moreover, the present study did not include any minors.

\section{Patient consent for publication}

Not applicable.

\section{Competing interests}

The authors declare that they have no competing interests.

\section{References}

1. Mac Grogan G, Collins LC, Lerwill M, Rakha EA and Tan BY: Solid papillary carcinoma (in situ and invasive). In: WHO Classification of Tumours. 5th edition. Breast Tumours IARC, Lyon, pp63-65, 2019.

2. Otsuki Y, Yamada M, Shimizu S, Suwa K, Yoshida M, Tanioka F, Ogawa H, Nasuno H, Serizawa A and Kobayashi H: Solid-papillary carcinoma of the breast: Clinicopathological study of 20 cases. Pathol Int 57: 421-429, 2007.

3. Goto Y, De Silva MG, Toscani A, Prabhakar BS, Notkins AL and Lan MS: A novel human insulinoma-associated cDNA, IA-1, encodes a protein with 'zinc-finger' DNA-binding motifs. J Biol Chem 267: 15252-15257, 1992.

4. Gierl MS, Karoulias N, Wende H, Strehle M and Birchmeier C: The zinc-finger factor Insm1 (IA-1) is essential for the development of pancreatic beta cells and intestinal endocrine cells. Genes Dev 20: 2465-2478, 2006.

5. Lan MS and Breslin MB: Structure, expression, and biological function of INSM1 transcription factor in neuroendocrine differentiation. FASEB J 23: 2024-2033, 2009.
6. Jia S, Wildner $\mathrm{H}$ and Birchmeier C: Insm1 controls the differentiation of pulmonary neuroendocrine cells by repressing Hes1. Dev Biol 408: 90-98, 2015.

7. Rooper LM, Bishop JA and Westra WH: INSM1 is a sensitive and specific marker of neuroendocrine differentiation in head and neck tumors. Am J Surg Pathol 42: 665-671, 2018.

8. Seok JY, Kang M, De Peralta-Venturina M and Fan X: Diagnostic utility of INSM1 in medullary thyroid carcinoma. Int J Surg Pathol 29: 615-626, 2021.

9. Dermawan JK and Mukhopadhyay S: Insulinoma-associated protein 1 (INSM1) differentiates carcinoid tumourlets of the lung from pulmonary meningothelial-like nodules. Histopathology 72 : 1067-1069, 2018.

10. Sakakibara R, Kobayashi M, Takahashi N, Inamura K, Ninomiya H, Wakejima R, Kitazono S, Yanagitani N, Horiike A, Ichinose $\mathbf{J}$, et al: Insulinoma-associated Protein 1 (INSM1) is a better marker for the diagnosis and prognosis estimation of small cell lung carcinoma than neuroendocrine phenotype markers such as chromogranin A, synaptophysin, and CD56. Am J Surg Pathol 44: 757-764, 2020

11. Mukhopadhyay S, Dermawan JK, Lanigan CP and Farver CF: Insulinoma-associated protein 1 (INSM1) is a sensitive and highly specific marker of neuroendocrine differentiation in primary lung neoplasms: An immunohistochemical study of 345 cases, including 292 whole-tissue sections. Mod Pathol 32: 100-109, 2019.

12. González I, Lu HC, Sninsky J, Yang C, Bishnupuri K, Dieckgraefe B, Cao D and Chatterjee D: Insulinoma-associated protein 1 expression in primary and metastatic neuroendocrine neoplasms of the gastrointestinal and pancreaticobiliary tracts. Histopathology 75: 568-577, 2019.

13. Tanigawa M, Nakayama M, Taira T, Hattori S, Mihara Y, Kondo R, KusanoH,Nakamura K,Abe Y,Ishida Y, et al:Insulinoma-associated protein 1 (INSM1) is a useful marker for pancreatic neuroendocrine tumor. Med Mol Morphol 51: 32-40, 2018.

14. Kudo N, Takano J, Kudoh S, Arima N and Ito T: INSM1 immunostaining in solid papillary carcinoma of the breast. Pathol Int 71: 51-59, 2021.

15. Seijnhaeve E, Galant C and Van Bockstal MR: Nuclear insulinoma-associated Protein 1 expression as a marker of neuroendocrine differentiation in neoplasms of the breast. Int J Surg Pathol 29: 496-502, 2021.

16. Metovic J, Castellano I, Marinelli E, Osella-Abate S, Sapino A, Cassoni P and Papotti M: INSM1 expression in breast neoplasms with neuroedocrine features. Endocr Pathol 32: 452-460, 2021.

17. Jiao Y, Shi C, Edil BH, de Wilde RF, Klimstra DS, Maitra A, Schulick RD, Tang LH, Wolfgang CL, Choti MA, et al: DAXX/ ATRX, MEN1, and mTOR pathway genes are frequently altered in pancreatic neuroendocrine tumors. Science 331: 1199-1203, 2011.

18. Marinoni I, Kurrer AS, Vassella E, Dettmer M, Rudolph T, Banz V, Hunger F, Pasquinelli S, Speel EJ and Perren A: Loss of DAXX and ATRX are associated with chromosome instability and reduced survival of patients with pancreatic neuroendocrine tumors. Gastroenterology 146: 453-460. e5, 2014.

19. Wang F, Xu X, Ye Z, Qin Y, Yu X and Ji S: Prognostic significance of altered ATRX/DAXX gene in pancreatic neuroendocrine tumors: A meta-analysis. Front Endocrinol (Lausanne) 12: 691557, 2021.

20. Matsuo K, Taniguchi K, Hamamoto H, Inomata Y, Komura K, Tanaka T, Lee SW and Uchiyama K: Delta-like canonical Notch ligand 3 as a potential therapeutic target in malignancies: A brief overview. Cancer Sci 112: 2984-2992, 2021.

21. Elston CW and Ellis IO: Pathological prognostic factors in breast cancer. I. The value of histological grade in breast cancer: Experience from a large study with long-term follow-up. C.W. Elston \& I O Ellis Histopathology 1991; 19.403-410. Histopathology 41 (3A): 151-152, discussion 152-3, 2002.

22. Razvi H, Tsang JY, Poon IK, Chan SK, Cheung SY, Shea KH and Tse GM: INSM1 is a novel prognostic neuroendocrine marker for luminal B breast cancer. Pathology 53: 170-178, 2021.

23. Vranic S, Palazzo J, Sanati S, Florento E, Contreras E, Xiu J, Swensen $J$ and Gatalica Z: Potential novel therapy targets in neuroendocrine carcinomas of the breast. Clin Breast Cancer 19: 131-136, 2019.

This work is licensed under a Creative Commons Attribution-NonCommercial-NoDerivatives 4.0 International (CC BY-NC-ND 4.0) License. 